\title{
Hidden quasi-symmetries stabilize non-trivial quantum oscillations in CoSi
}

\section{Chunyu Guo ( $\nabla$ chunyu.guo@epfl.ch )}

Ecole Polytechnique Federale de Lausanne (EPFL) https://orcid.org/0000-0001-8339-7477

\section{Lunhui Hu}

The Pennsylvania State University

\section{Carsten Putzke}

Laboratory of Quantum Materials https://orcid.org/0000-0002-6205-9863

\section{Jonas Diaz}

Ecole Polytechnique Federale de Lausanne (EPFL)

\section{Xiangwei Huang}

Ecole Polytechnique Federale de Lausanne (EPFL)

\section{Kaustuv Manna}

Max Planck Institute for Chemical Physics of Solids https://orcid.org/0000-0002-9442-2321

\section{Feng-Ren Fan}

Max Planck Institute for Chemical Physics of Solids

\section{Chandra Shekhar}

Max Planck Institute for Chemical Physics of Solids https://orcid.org/0000-0002-3330-0400

\section{Yan Sun}

Max Planck Institute for Chemical Physics of Solids https://orcid.org/0000-0002-7142-8552

\section{Claudia Felser}

Max Planck Institute for Chemical Physics of Solids https://orcid.org/0000-0002-8200-2063

Chao-Xing Liu

Pennsylvania State University https://orcid.org/0000-0003-1881-1365

\section{Bogdan Bernevig}

Princeton University https://orcid.org/0000-0001-6337-4024

\section{Philip Moll}

École Polytechnique Fédérale de Lausanne https://orcid.org/0000-0002-7616-5886

\section{Article}

Keywords: topological matter, quasi-symmetries, quantum oscillations, CoSi

Posted Date: September 7th, 2021 
DOI: https://doi.org/10.21203/rs.3.rs-811950/v1

License: (c) (1) This work is licensed under a Creative Commons Attribution 4.0 International License. Read Full License

Version of Record: A version of this preprint was published at Nature Physics on May 16th, 2022. See the published version at https://doi.org/10.1038/s41567-022-01604-0. 


\title{
Hidden quasi-symmetries stabilize non-trivial quantum oscillations in $\mathrm{CoSi}$
}

\author{
Chunyu Guo, ${ }^{1 *}$ Lunhui Hu, ${ }^{2 *}$ Carsten Putzke, ${ }^{1}$ Jonas Diaz, ${ }^{1}$ Xiangwei \\ Huang, ${ }^{1}$ Kaustuv Manna, ${ }^{3,4}$ Feng-Ren Fan, ${ }^{3}$ Chandra Shekhar, ${ }^{3}$ Yan Sun, ${ }^{3}$ \\ Claudia Felser, ${ }^{3}$ Chaoxing Liu, ${ }^{2 \dagger}$ B. Andrei Bernevig, ${ }^{5,6,7 \dagger}$ Philip J. W. Moll ${ }^{1 \dagger}$ \\ ${ }^{1}$ Laboratory of Quantum Materials (QMAT), Institute of Materials (IMX), \\ École Polytechnique Fédérale de Lausanne (EPFL), CH-1015 Lausanne, Switzerland \\ ${ }^{2}$ Department of Physics, The Pennsylvania State University, University Park, PA, USA \\ ${ }^{3}$ Max Planck Institute for Chemical Physics of Solids, 01187 Dresden, Germany \\ ${ }^{4}$ Department of Physics, Indian Institute of Technology Delhi, New Delhi 110016, India \\ ${ }^{5}$ Department of Physics, Princeton University, Princeton, New Jersey 08544, USA \\ ${ }^{6}$ Donostia International Physics Center,P. Manuel de Lardizabal 4, 20018 \\ Donostia-San Sebastian, Spain \\ ${ }^{7}$ IKERBASQUE, Basque Foundation for Science, Bilbao, Spain
}

${ }^{*}$ These authors contributed equally to this work.

${ }^{\dagger}$ Corresponding authors: cxl56@psu.edu(C.X.L.); bernevig@ princeton.edu(B.A.B.); philip.moll@epfl.ch(P.J.W.M.)

Unlocking the exotic properties promised to occur in topologically non-trivial semi-metals currently requires significant fine-tuning. Crystalline symmetry restricts the location of topological defects to isolated points (0D) or lines (1D), as formalized by the Wigner-Von Neumann theorem. The scarcity of materials in which these anomalies occur at the chemical potential is a major obstacle towards their applications. Here we show how non-crystalline quasisymmetries stabilize near-degeneracies of bands over extended regions in en- 
ergy and in the Brillouin zone. Specifically, a quasi-symmetry is an exact symmetry of a $k \cdot p$ Hamiltonian to lower-order that is broken by higher-order terms. Hence quasi-symmetric points are gapped, yet the gap is parametrically small and therefore does not influence the physical properties of the system. We demonstrate that in the eV-bandwidth semi-metal CoSi an internal quasisymmetry stabilizes gaps in the $\mathbf{1 - 2} \mathbf{m e V}$ range over a large near-degenerate plane (2D). This quasi-symmetry is key to explaining the surprising simplicity of the experimentally observed quantum oscillations of four interpenetrating Fermi surfaces around the R-point. Untethered from the limitations of crystalline symmetry, quasi-symmetries eliminate the need for fine-tuning as they enforce sources of large Berry curvature to occur at the chemical potential, and thereby lead to new Wigner-Von Neumann classifications of solids. Quasisymmetries arise from a comparable splitting of degenerate states by spinorbit coupling and by orbital dispersion - suggesting a hidden classification framework for symmetry groups and materials in which quasi-symmetries are critical to understand the low-energy physics.

The introduction of concepts of topology in the past years into the field of electronic dispersions has captured the imagination of condensed matter physics and sparked a flurry of new research directions in the past years. At its core is the realization that the electronic waveforms constituting a material cannot always be derived from an entirely local description. In these topologically non-trivial metals, the wavefunctions are inherently non-local, and as such novel electronic states are expected and observed at the crystal surface terminating them (1-10). Beyond these surface properties, interesting anomalies in the bulk dispersion lead to novel physical phenomena, such as the emergence of quasiparticles mimicking ultra-relativistic Weyl- and Dirac-Fermions in 3D; a solid-state analogon of the Adler-Bell-Jackiw anomaly; a planar Hall 
effect; topological piezoelectric effect; second harmonic generation in Weyl semimetals; or strong Berry curvature that impacts the semi-classical and quantum dynamics of quasiparticles $(11-19)$.

Given the wealth of new phenomena and potential applications, it is important to identify such "ideal" Weyl-, Dirac- or nodal-line semi-metals in which a priori unrelated chemical potential and the topological anomaly coincide. That such a coincidence is rather rare can be understood from the Wigner-Von Neumann theorem, which states that a two-level crossing generally requires three tuning parameters $(24,25)$. Any two-level model can be expanded as $H=\epsilon_{0}+\sum_{i} d_{i} \sigma_{i}$ where $i=x, y, z$ and $\epsilon_{0}$ is an offset, while the momenta $k_{i}$ take the role of the generic tuning parameters $d_{i}$ in this discussion. Thus to obtain a two level degeneracy, all 3 tuning parameters are required to achieve $d_{x}=d_{y}=d_{z}=0$ (codimension 3). Such a point would generally only coincide with the Fermi level accidentally. To stabilize crossings of more than two bands or achieve higher dimensional degeneracies (nodal lines, planes), additional crystalline symmetries must provide further constraints on the form of the Hamiltonian, such as two-fold screw axis for the nodal plane in $\mathrm{MnSi}$ (26) or rotational symmetries for the fourfold Dirac points in the 3D Dirac semi-metals $\mathrm{Cd}_{3} \mathrm{As}_{2}$ and $\mathrm{Na}_{3} \mathrm{Bi}(6,7)$. As symmetry and topology are inseparably intertwined, it is natural that symmetry should guide our search for interesting materials.

Here we refine this picture, and argue for the importance of approximate symmetries as an additional concept [Fig. 1(a)] that can generate interesting measurable quantum phenomena. A crystalline symmetry operation, $M$, commutes with the Hamiltonian of the system, $[H(k), M]=0$. The well-known representation theory dictates that low-symmetry points, such as generic k-point in the Brilloiun zone, usually support only one-dimensional representations, and hence bands anti-cross at these points. It may further be possible, however, that at these low symmetry points approximate symmetries emerge and can even be k-dependent. They are 
not exact symmetries of the material yet still commute with a dominant part of the Hamiltonian. Such approximate symmetries would stabilize almost linear band crossings by enforcing finite yet perturbatively small energy gaps. They already naturally occur when exact symmetries are weakly broken, such as tiny monoclinic distortions weakly removing a rotational symmetry that otherwise stabilizes a band crossing point. Graphene could be considered as an alternative example in the spin channel, as its Dirac point is weakly gapped when spin-orbit coupling is considered, turning spin-rotation into an approximate symmetry (27). However, as approximate symmetries leave the bounds set by crystalline point-groups, more exciting hidden approximate symmetries may emerge that have no counterpart in the crystal symmetry. A famous example of a hidden symmetry is the Laplace-Runge-Lenz vector in classical gravitation. It is the conserved quantity stabilized by the hidden $\mathrm{SO}(4)$ symmetry of the $r^{-1}$ potential of the Kepler problem that enforces strictly closed classical gravitational orbits. Weak perturbations, such as $r^{-1+\epsilon}$, mathematically reduce the symmetry to $\mathrm{SO}(3)$, yet the almost closed orbits for small $\epsilon$ evidence that the system senses the proximity to that hidden symmetry.

We adopt the term "quasi-symmetry" to denote a special type of approximate symmetry of electronic band structures, that emerges from the hierarchy of a $k \cdot p$ type of perturbation expansion around a high-symmetry point. Quasi-symmetries are operators $M_{\text {eff }}$ that are the exact symmetries of the lower-order expansion but not of higher-order perturbation terms. The main goal of this paper is to show such a quasi-symmetry exists in the semi-metal CoSi as well as a large set of similar materials, and that it enforces a low-symmetry plane of neardegeneracies spanning the Brillouin zone which pin a source of Berry curvature to the Fermi level (Fig. 1). Excitingly, it falls into the latter category of hidden quasi-symmetries that are unrelated to approximate point-group symmetries, but can be treated as a symmetry of internal degrees of freedom, akin to spin in graphene. Unlike graphene, however, the form of quasisymmetry is non-trivial as the corresponding symmetry operator is k-dependent and forms a 
part of the Hamiltonian. It has remarkable consequences on the quantum oscillation spectrum, which we here present as the first experimental observation of material properties critically defined by quasi-symmetries.

CoSi crystallizes in a chiral cubic structure of space group $P 2{ }_{1} 3$ without an inversion center [Fig. 2(a)]. Recent studies revealed the existence of exceptionally long Fermi arcs which connect the sixfold and fourfold band degenerate points $(9,29-31)$. These two degenerate points correspond to two branches of Fermi surfaces located at at the $\Gamma$ - and R-point, respectively, yet only the R-point pockets are observed in quantum oscillation experiments. Band structure calculations reveal four split bands due to spin-orbit coupling which correspond to four Fermi surfaces at $\mathrm{R}$, which we label as $1^{+}, 1^{-}, 2^{+}, 2^{-}$, representing the orbital and spin degree of freedom respectively. States at the boundary of the Brillouin zone are doubly degenerate due to crystalline symmetry, hence the non-degenerate Fermi surfaces are enforced to touch there (5). The spin-orbit coupling and the orbital gap are both small yet similar in magnitude, which explains how four slightly degenerate, interpenetrating spheroids make up a surprisingly rich internal structure of the Fermi surfaces.

CoSi single crystals resemble octahedra, indicating a dominant growth along the [111] direction (see supplement for growth details). The quality of the single-phase crystallinity was checked by Laue and single crystal X-Ray diffraction. These crystals were fabricated into microstructures using focused-ion-beam machining (32) (see supplement) to increase the current path homogeneity and signal of Shubnikov-de Haas oscillations. The resistivity as well as the large residual resistivity ratio in the samples agrees well with previous bulk measurements (34), indicating an unchanged material quality during fabrication (Fig. 3). Hence not surprisingly, large quantum oscillations of the magnetoresistance are readily observed at low fields. Subtracting a polynomial background uncovers strong oscillations that resemble a beating pattern of two frequencies around $F_{1} \sim 550 \mathrm{~T}$ and $F_{2} \sim 660 \mathrm{~T}$. These two, and their harmonics, are the 
only frequencies observed in our as well as previous experiments (33-36). The temperature dependence of the oscillations follows well the Lifshitz-Kosevich form, leading to a low cyclotron effective mass of $m_{c} \sim 0.84 m_{e}$.

In light of the complexity of the self-intersecting Fermi surfaces it appears at first surprising that the quantum oscillation spectrum is tantalizingly simple, with only two frequences that, most importantly, only weakly depend on the direction of the applied magnetic field (Fig. 4). When magnetic quantum oscillations arise from such highly degenerate Fermi surfaces, the main question is which, if any, possible trajectories become quantum coherent. As the Fermisurface is centered around the R-point, any orbit necessarily crosses the symmetry-enforced degeneracies at the Brillouin zone boundary multiple times. This has been argued to stabilize band degeneracies pinned to the Fermi level in this crystal structure $(26,36)$ on the planes of Brilloiun zone boundary [marked pink in Fig. 4(a)] .

Yet the orbits further intersect at low-symmetry points at which a gap must open [marked blue in Fig. 4(a)]. Neither the frequencies nor their angle dependence obtained from DFT calculations match the data if this gap is considered to separate the orbits via significant avoidedcrossing. As the gap is small, the quasiparticle may tunnel across it continuing on the original trajectory, a phenomenon known as magnetic breakdown (37-40). Based on estimations of the gap from DFT and further analytical calculations, vanishingly small breakdown fields fall below $0.11 \mathrm{~T}$ for any field orientation, and hence the experimental data is always obtained in an extreme breakdown regime (see supplement for details). Thus the wavefunctions propagate through these points at perfect transmission, and indeed an excellent match of frequencies and their angle dispersion between theory and experiment is found (Fig. 4).

The oscillation spectrum thus can be completely rationalized a priori without quasi-symmetries. However one overlooks a key question about the structure of the wavefunctions, namely why in a metal with $\mathrm{eV}$ bandwidth two bands anti-cross with a gap no larger than $2 \mathrm{meV}$ over the 
entire Fermi surface. To obtain such a parametrically small gap accidentally, an unreasonable degree of fine-tuning is required that acts simultaneously at many k-points, as well as at a wide range of energies as we will show. Instead, a hidden quasi-symmetry enforces the smallness of the gap at these points, and thus explains why magnetic breakdown at full transparency occurs at any arbitrary angle.

To understand this quasi-symmetry, we next consider an effective model around the R-point. Without spin-orbit coupling (SOC), all the bands are eight-fold degenerate at $\mathrm{R}$ due to the combination of two-fold screw axis symmetries along the $\mathrm{x}$ and $\mathrm{y}$ direction and time reversal symmetry. Including spin doubles the degeneracy and the SOC splits the eight-fold degenerate states into six-fold and two-fold degenerate states at the R-point. An effective model for these eight bands around the R-point can be constructed as

$$
\mathcal{H}_{R}=\mathcal{H}_{0}(\mathbf{k})+\mathcal{H}_{s o c}+\mathcal{H}_{k^{2}}(\mathbf{k})
$$

where $\mathcal{H}_{0}(\mathbf{k})=C_{0}+2 A_{1}(\mathbf{k} \cdot \mathbf{L})$ is the lower-order expansion of the spin-independent Hamiltonian, and three 4-by-4 matrices $\mathbf{L}$ form an emergent angular momentum algebra $\left[L_{i}, L_{j}\right]=$ $i \varepsilon_{i j k} L_{k}$ with Levi-Civita symbol $\varepsilon_{i j k}$ and $i, j, k=x, y, z$. The SOC term is given by $\mathcal{H}_{s o c}=$ $2 \lambda_{0}(\mathbf{s} \cdot \mathbf{L})$, where $\mathbf{s}$ is the spin operator. $\mathcal{H}_{k^{2}}(\mathbf{k})$ is the higher-order spin-independent term with its form given in the supplement. Here $\mathcal{H}_{0}(k)+\mathcal{H}_{k^{2}}(k)$ is the SOC-free expanded Hamiltonian to second order. By choosing appropriate parameters such as $C_{0}, A_{1}, \lambda_{0}$, the energy dispersion of $\mathcal{H}_{R}$ well reproduces that from the DFT calculations (see supplement).

A striking feature in the Hamiltonian $\mathcal{H}_{R}$ is that the $\mathrm{SOC}$ term $\mathcal{H}_{s o c}$ takes a similar form as the linear-momentum term, just by replacing the momentum $\mathbf{k}$ by spin $\mathbf{s}$. This is because spin, as a pseudo-vector, behaves exactly the same as a vector due to the lack of inversion, mirror or other roto-inversion symmetries for a chiral crystal. Due to this similarity, up to the first-order perturbation, we find that the spin of the eigen-states is parallel or anti-parallel to the momen- 
tum $\mathrm{k}$ and thus we can label the spin states of these bands by \pm in Fig. 1(e). We derive an effective Hamiltonian for the near degenerate bands. This can be done by first projecting the full Hamiltonian $\mathcal{H}_{R}$ into the 4 closest bands $\left(1^{ \pm}\right.$and $2^{ \pm}$bands) to get a 4-band model and then constructing a 2-band model for the $1^{-}$and $2^{+}$bands, as discussed in supplement. Up to the first-order perturbation, the 2-band Hamiltonian takes the form $H_{\text {eff }}=\epsilon_{0}+d_{z}(\mathbf{k}) \sigma_{z}$, where $d_{z}(\mathbf{k})=\lambda_{0}-\sqrt{3} \tilde{C} \frac{\left|k_{x} k_{y} k_{z}\right|}{k}$ and the form of the function $\epsilon_{0}$ is given in supplement. Here $\sigma_{z}$ is the Pauli matrix on the basis of $1^{-}$and $2^{+}$bands, $\lambda_{0}$ term comes from the SOC Hamiltonian $\mathcal{H}_{s o c}$ and the $\tilde{C}$ term from $\mathcal{H}_{k^{2}}(\mathbf{k})$. It is striking to see that the operator $\mathcal{M}_{e f f}=\sigma_{z}$ commutes with $H_{\text {eff }}$ and thus serves as an exact symmetry at the first-order expansion. The existence of $\mathcal{M}_{\text {eff }}$ forbids any terms that are coupled to $\sigma_{x, y}$ in the effective Hamiltonian, thus reducing the codimension of a two-level crossing from 3 to 1 and stabilizing a nodal plane, which is defined by the equation $\lambda_{0}=\sqrt{3} \tilde{C} \frac{\left|k_{x} k_{y} k_{z}\right|}{k}$ in this two-band model. A more accurate and complete description around the Fermi energy is the 4-band model, which shares a similar k-dependent hidden quasi-symmetry and is essential in deriving the 2-band model (see supplement), and the whole perturbation theory reveals a striking hierarchy structure (41). Higher-order perturbation expansion breaks $\mathcal{M}_{\text {eff }}$ through inducing additional terms coupled to $\sigma_{x, y}$ in the effective model and thus leads to a small gap opening of this nodal plane (See supplement). Therefore, $\mathcal{M}_{\text {eff }}$ is not a symmetry of the system, but an emergent quasi-symmetry in the sense of low-energy effective theory. Importantly, this curved nodal plane spans low-symmetry regions of the Brillouin zone, in contrast to symmetry-enforced exact degeneracies. It is this combined structure of symmetry and quasi-symmetry induced degeneracies that forms the basis of the complex structure of the Fermi surface centered at $\mathrm{R}$, and reduces the complexity of the quantum orbits in magnetic fields to two, angle-independent frequencies.

Compared to the exact degeneracy due to symmetry, near degeneracies from quasi-symmetry have unique implications in the Berry curvature distribution in momentum space [Fig. 1(e)]. 
The U(1) Berry curvature is not well-defined at a degeneracy, while for near-degeneracy, the small gap concentrates significant Berry curvature. In our model, the Berry curvature almost vanishes in the vicinity of the exact degenerate plane, yet it is concentrated to a ring on the near nodal plane at any fixed energy. This large Berry curvature around the near nodal lines, which always occurs at the Fermi energy, may strongly affect physical phenomena that are related to local Berry curvature, including intrinsic spin Hall effect $(42,43)$ and quantum nonlinear Hall effect (44). The strong Berry curvature is focused into the near-degeneracy, where the quasiparticle tunneling occurs. It will be interesting to explore how this Berry curvature modifies the tunneling conditions in magnetic breakdowns at such quasi-symmetry given neardegeneracies (45).

The observation of quasi-symmetries raises new questions on how wave functions can be woven together in condensed matter systems. The most practical concerns finding the main ingredients for quasi-symmetries to occur in actual materials. The novelty of separating them from crystalline symmetries equally presents a challenge in that a detailed and rigorous analysis of the electronic bands at all low-symmetry k-points is required to identify them. Search programs based on symmetry analysis will not find this type of anomaly without further guideline. It is natural, however, to suspect related compounds crystallizing in the same structure to share this feature, such as e.g. PtGa, PtAl, RhSi and the magnetic MnSi where the influence of magnetism can be explored. $(26,46-54)$. At the same time, it raises the interesting prospect that even those materials in which crystalline symmetries do not allow stable band crossings may still host exotic quasiparticles and strong Berry curvature at the Fermi level due to the hidden proximity of the system to a topological state. Here, quantum oscillation experiments may provide guidance by pointing to materials with similarly extreme magnetic breakdown on extended regions on the Fermi surface, which a quasi-symmetry would naturally explain without further fine-tuning. On the theoretical side, it will be critical to elucidate further the physical meaning 
of the $M_{\text {eff }}$ in $\mathrm{CoSi}$ and to evolve the understanding of the mathematical structure behind it. These developments are expected to hold an interesting future for the role of topology. A consensus of high-throughput efforts emerges that 20-30\% of materials are topological (20-23). Yet it appears that crystal symmetry may not hold up as the strict barrier it was thought to be, and via quasi-symmetries concepts of topology may reach even further than these estimates predict.

\section{References}

1. Armitage, N. P., Mele, E. J., and Vishwanath, A. Weyl and Dirac semimetals in threedimensional solids. Rev. Mod. Phys. 90, 015001 (2018).

2. Lv, B. Q., Qian, T., and Ding, H. Experimental perspective on three-dimensional topological semimetals. Rev. Mod. Phys. 93, 025002 (2021).

3. Wan, X. G., Turner, A. M., Vishwanath, A., and Savrasov, S. Y. Topological semimetal and Fermi-arc surface states in the electronic structure of pyrochlore iridates. Phys. Rev. B 83, $205101(2011)$.

4. Wang, Z., Alexandradinata, A., Cava, R. J., and Bernevig, B. A. Hourglass fermions. Nature 532, $7598(6)$.

5. Bradlyn, B., Cano, J., Wang, Z., Vergniory, M. G., Felser, C., Cava, R. J., and Bernevig, B. A. Beyond Dirac and Weyl fermions: Unconventional quasiparticles in conventional crystals. Science 353, aaf5037 (2016).

6. Wang, Z., Sun, Y., Chen, X.-Q., Franchini, C., Xu, G., Weng, H., Dai, X., and Fang, Z. Dirac semimetal and topological phase transitions in $\mathrm{A}_{3} \mathrm{Bi}(\mathrm{A}=\mathrm{Na}, \mathrm{K}, \mathrm{Rb})$. Phys. Rev. $\mathrm{B}$ 85, 195320 (2012). 
7. Wang, Z., Weng, H., Wu, Q., Dai, X., and Fang, Z. Three-dimensional Dirac semimetal and quantum transport in $\mathrm{Cd}_{3} \mathrm{As}_{2}$. Phys. Rev. B 88, 125427 (2013).

Titus

8. Bian, G., Chang, T., Zheng, H., Velury, S., Xu, S-. Y., and Neupert, T. et al., Drumhead surface states and topological nodal-line fermions in TlTaSe 2 . Phys. Rev. B 93, 121113 (2016).

9. Rao, Z., Li, H., Zhang, T., Tian, S., Li, C., and Fu, B. et al., Observation of unconventional chiral fermions with long Fermi arcs in CoSi. Nature 567, 496 (2019).

10. Moll, P. J. W., Nair, N. L., Helm, T., Potter, A. C., Kimchi, I., Vishwanath, A., and Analytis, J. G. Transport evidence for Fermi-arc-mediated chirality transfer in the Dirac semimetal $\mathrm{Cd}_{3} \mathrm{As}_{2}$. Nature 535, 266 (2016).

11. Zyuzin, A. A., and Burkov, A. A. Topological response in Weyl semimetals and the chiral anomaly. Phys. Rev. B 86, 115133 (2012).

12. Burkov, A. A. Chiral anomaly and transport in Weyl metals. J. Condens. Matter Phys. 27, $113201(2015)$.

13. Xiong, J., Kushwaha, S. K., Liang, T., Krizan, J. W., Hirschberger, M., and Wang, W. et al., Evidence for the chiral anomaly in the Dirac semimetal $\mathrm{Na}_{3} \mathrm{Bi}$. Science 350, 413 (2015).

14. Matsushita, T., Fujimoto, S., and Schnyder, A. P. Topological piezoelectric effect and parity anomaly in nodal line semimetals. Phys. Rev. Research 2, 043311 (2020).

15. Li, Z., Jin, Y-. Q., Tohyama, T., Iitaka, T., Zhang, J-. X., and Su, H. Second harmonic generation in the Weyl semimetal TaAs from a quantum kinetic equation. Phys. Rev. B 97, 085201 (2018). 
16. Takasan, K., Morimoto, T., Orenstein, J., and Moore, J. E. Current-induced second harmonic generation in inversion-symmetric Dirac and Weyl semimetals. arXiv: 2007.08887 (2020).

17. Burkov, A. A. Giant planar Hall effect in topological metals. Phys. Rev. B 96, 041110 (2017).

18. Liu, E., Sun, Y., Kumar, N., Muechler, L., Sun, A., and Jiao, L. et al., Giant anomalous Hall effect in a ferromagnetic kagome-lattice semimetal. Nat. Phys. 14, 1125 (2018).

19. Nakatsuji, S., Kiyohara, N., and Higo, T. Large anomalous Hall effect in a non-collinear antiferromagnet at room temperature. Nature 527, 212 (2015).

20. Bradlyn, B., Elcoro, L., Cano, J., Vergniory, M., Wang, Z., Felser, C., Aroyo, M., and Bernevig, B. A. Topological quantum chemistry. Nature 547, 298 (2017).

21. Zhang, T., Jiang, Y., Song, Z., Huang, H., He, Y., Fang, Z., Weng, H., and Fang, C. Catalogue of topological electronic materials. Nature 566, 475 (2019).

22. Vergniory, M. G., Elcoro, L., Felser, C., Bernevig, B. A., and Wang, Z. A complete catalogue of high-quality topological materials. Nature 566, 480 (2019).

23. Tang, F., Po, H. C., Vishwanath, A., and Wan, X. G. Comprehensive search for topological materials using symmetry indicators. Nature 566, 486 (2019).

24. von Neumann, V. J., and Wigner, E., Uber merkwürdige diskrete Eigenwerte. Phys. Z. 30, 465 (1929).

25. Murakami, S., Iso, S., Avishai, Y., Onoda, M., and Nagaosa, N. Tuning phase transition between quantum spin Hall and ordinary insulating phases. Phys. Rev. B 76, 205304 (2007). 
26. Wilde, M. A., Dodenhöft, M., Niedermayr, A., Bauer, A., Hirschmann, M. M., and Alpin, K. et al., Symmetry-enforced topological nodal planes at the Fermi surface of a chiral magnet. Nature 594, 374 (2021).

27. Konschuh, S., Gmitra, M., and Fabian, J. Tight-binding theory of the spin-orbit coupling in graphene. Phys. Rev. B 82, 245412 (2010).

28. Sichau, J., Prada, M., Anlauf, T., Lyon, T. J., Bosnjak, B., Tiemann, L., and Blick, R. H. Resonance Microwave Measurements of an Intrinsic Spin-Orbit Coupling Gap in Graphene: A Possible Indication of a Topological State. Phys. Rev. Lett. 122, 046403 (2019).

29. Yuan, Q. Q., Zhou, L., Rao, Z. C., Tian, S., Zhao, W. M., and Xue, C. L. et al., Quasiparticle interference evidence of the topological Fermi arc states in chiral fermionic semimetal CoSi. Science advances 5, eaaw9485 (2019).

30. Sanchez, D. S., Belopolski, I., Cochran, T. A., Xu, X., Yin, J-. X., and Chang, G. Q. et al., Topological chiral crystals with helicoid-arc quantum states. Nature 567, 500 (2019).

31. Fang, C., Gilbert, M. J., Dai, X., and Bernevig, B. A. Multi-Weyl topological semimetals stabilized by point group symmetry. Phys. Rev. Lett. 108, 266802 (2012).

32. Moll, P. J. W. Focused Ion Beam Microstructuring of Quantum Matter. Annu. Rev. Condens. Matter Phys. 9, 147 (2018).

33. Wu, D. S., Mi, Z. Y., Li, Y. J., Wu, W., Li, P. L., and Song, Y. T. et al., Single Crystal Growth and Magnetoresistivity of Topological Semimetal CoSi. Chin. Phys. Lett. 36, $077102(2019)$. 
34. Xu, X., Wang, X., Cochran, T. A., Sanchez, D. S., Chang, G., and Belopolski, I. et al., Crystal growth and quantum oscillations in the topological chiral semimetal CoSi. Phys. Rev. B 100, 045104 (2019).

35. Wang, H., Xu, S., Lu, X. Q., Wang, X. Y., Zeng, X. Y., and Lin, J. F. et al., de Haas-van Alphen quantum oscillations and electronic structure in the large-Chern-number topological chiral semimetal CoSi. Phys. Rev. B 102, 115129 (2020).

36. Huber, N., Alpin, K., Causer, G. L., Worch, L., Bauer, A., Benka, G. et al., Network of topological nodal planes, multifold degeneracies, and Weyl points in CoSi. arXiv 2107, $02820(2021)$.

37. Blount, E. I., Bloch Electrons in a Magnetic Field. Phys. Rev. 126, 1636 (1962).

38. Helm, T., Kartsovnik, M. V., Proust, C., Vignolle, B., Putzke, C., Kampert, E. et al., Correlation between Fermi surface transformations and superconductivity in the electron-doped high- $T_{c}$ superconductor $\mathrm{Nd}_{2-x} \mathrm{Ce}_{x} \mathrm{CuO}_{4}$. Phys. Rev. B 92, 094501 (2015).

39. Honold, M. M., Harrison, N., Nam, M.-S., Singleton, J., Mielke, C. H., Kurmoo, M., and Day, P. Magnetic breakdown in the high-field phase of the organic conductor $\alpha$-(BEDT$\mathrm{TTF})_{2} \mathrm{KHg}(\mathrm{SCN})_{4}$. Synth. Met. 103, 2093 (1999).

40. Pezzini, S., van Delft, M. R., Schoop, L. M., Lotsch, B. V., Carrington, A., Katsnelson, M. I., Hussey, N. E., and Wiedmann, S. et al., Unconventional mass enhancement around the Dirac nodal loop in ZrSiS. Nat. Phys. 14, 178 (2017).

41. Hu, L. H., Guo, C. Y., Putzke, C., Diaz, J., Huang, X. W., Manna, K., Fan, F. -R., Shekhar, C., Sun, Y., Felser, C., Moll, P. J. W., Liu, C. X., Bernevig, B. A. (unpublished). . 
42. Sun, Y., Zhang, Y., Liu, C. X., Felser, C., and Yan, B. Dirac nodal lines and induced spin Hall effect in metallic rutile oxides. Phys. Rev. B 95, 235104 (2017).

43. Sun, Y., Zhang, Y., Felser, C., and Yan, B. Strong intrinsic spin Hall effect in the TaAs family of Weyl semimetals. Phys. Rev. Lett. 117, 146403 (2016).

44. Sodemann, I. and Fu, L. Quantum Nonlinear Hall Effect Induced by Berry Curvature Dipole in Time-Reversal Invariant Materials. Phys. Rev. Lett. 115, 216806 (2015).

45. Wang, C., Duan, W., Glazman, L., and Alexandradinata, A. Landau quantization of nearly degenerate bands and full symmetry classification of Landau level crossings. Phys. Rev. B. 100, 014442 (2019).

46. Yao, M., Manna, K., Yang, Q., Fedorov, A., Voroshnin, V., and Schwarze, B. V. et al., Observation of giant spin-split Fermi-arc with maximal Chern number in the chiral topological semimetal PtGa. Nat. Comms. 11, 1 (2020).

47. Ma, J. Z., Wu, Q. S., Song, M., Zhang, S.-N., Guedes, E. B. and Ekahana, S. A. et al., Observation of a singular Weyl point surrounded by charged nodal walls in PtGa. Nat. Comms. 12, 3994 (2021).

48. Xu, S., Zhou, L., Wang, X.Y., Wang, H., Lin, J.F., Zeng, X.Y., Cheng, P., Weng, H. and Xia, T.L. Quantum Oscillations and Electronic Structure in the Large-Chern-Number Topological Chiral Semimetal PtGa. Chin. Phys. Lett. 37, 107504 (2020).

49. Schröter, N. B. M., Pei, D., Vergniory, M. G., Sun, Y, Manna, K., and de Juan, F. et al., Chiral topological semimetal with multifold band crossings and long Fermi arcs. Nat. Phys. 15, 579 (2019). 
50. Schröter, N. B. M., Stolz, S., Manna, K., de Juan, F., Vergniory, M. G., and Krieger J. A. et al., Observation and control of maximal Chern numbers in a chiral topological semimetal. Science 369, 179 (2020).

51. Sessi, P., Feng-ren, F., Küster, F., Manna, K., and Vergniory, M. G. et al., Handednessdependent quasiparticle interference in the two enantiomers of the topological chiral semimetal PdGa. Science 369, 179 (2020).

52. Chang, G., Xu, S. Y., Wieder, B. J., Sanchez, D. S., Huang, S. M., and Belopolski, I. et al., Unconventional chiral fermions and large topological Fermi arcs in RhSi. Phys. Rev. Lett. 119, 206401 (2017).

53. Rees, D., Manna, K., Lu, B., Morimoto, T., Borrmann, H., and Felser, C. et al., Helicitydependent photocurrents in the chiral Weyl semimetal RhSi. Sci. Adv. 6, eaba0509 (2020). A. Rosch, A.

54. Mühlbauer, S., Binz, B., Jonietz, F., Pfleiderer, C., Rosch, A., and Neubauer, A. et al., Skyrmion Lattice in a Chiral Magnet. Science 323, 915 (2009). 


\section{Acknowledgements:}

Funding: This work was funded by the European Research Council (ERC) under the European Union's Horizon 2020 research and innovation programme (MiTopMat - grant agreement No. 715730). This project received funding by the Swiss National Science Foundation (Grants No. PP00P2_176789). C. X. Liu and L. H. Hu are supported by the Office of Naval Research (Grant No. N00014-18-1-2793) and Kaufman New Initiative research Grant No. KA2018-98553 of the Pittsburgh Foundation. K.M., and C.F. acknowledge the financial support from the European Research Council (ERC) Advanced Grant No. 742068 "TOP-MAT"; European Union's Horizon 2020 research and innovation program (Grant Nos. 824123 and 766566)and Deutsche Forschungsgemeinschaft (DFG) through SFB 1143. Additionally, K. M. acknowledges Max Plank Society for the funding support under Max Plank-India partner group project. B.A.B. thanks funding from the European Research Council (ERC) under the European Union's Horizon 2020 research and innovation programme (grant agreement no. 101020833). B.A.B. is also supported by the U.S. Department of Energy (Grant No. DE-SC0016239), and partially supported by the National Science Foundation (EAGER Grant No. DMR 1643312), a Simons Investigator grant (No. 404513), the Office of Naval Research (ONR Grant No. N00014-201-2303), the Packard Foundation, the Schmidt Fund for Innovative Research, the BSF Israel US foundation (Grant No. 2018226), the Gordon and Betty Moore Foundation through Grant No. GBMF8685 towards the Princeton theory program, and a Guggenheim Fellowship from the John Simon Guggenheim Memorial Foundation. B.A.B. is supported by the NSF-MERSEC (Grant No. MERSEC DMR 2011750). B.A.B. gratefully acknowledge financial support from the Schmidt DataX Fund at Princeton University made possible through a major gift from the Schmidt Futures Foundation. B.A.B. received additional support from the Max Planck Society. Further support was provided by the NSF-MRSEC No. DMR-1420541, BSF Israel US foundation No. 2018226, and the Princeton Global Network Funds. 
377 Competing Interests The authors declare that they have no competing financial interests. 
(a)

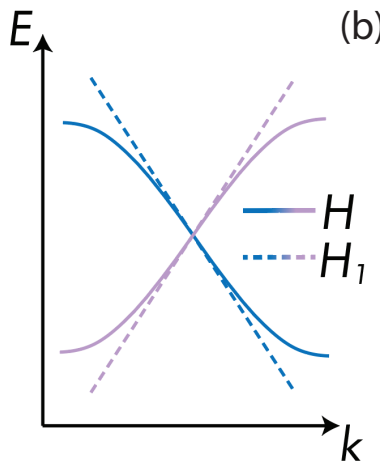

$[H, M]=0$

$H=H_{0}+\sum^{i} H_{\text {pert. }}^{(\mathfrak{i})}$ $H_{1}=H_{0}+H_{\text {pert. }}^{(1)}$. (b) $E$

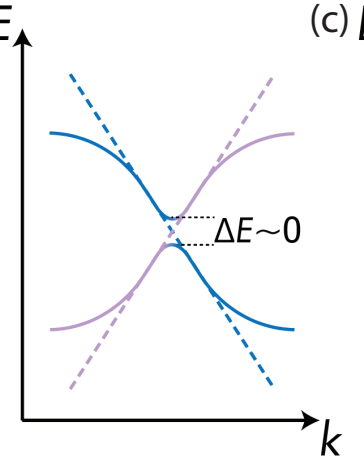

$\left[H, M_{\text {eff }}\right] \neq 0$

$\left[H_{1}, M_{\text {eff }}\right]=0$ (c)

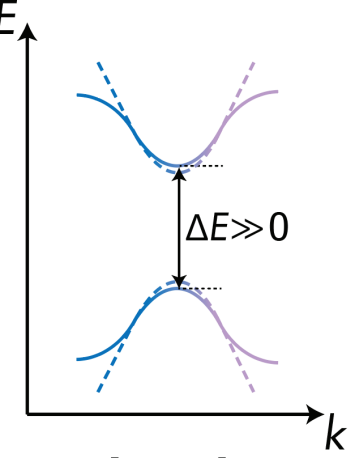

$\left[H, M_{\text {eff }}\right] \neq 0$

$\left[H_{1}, M_{\text {eff }}\right] \neq 0$

(d)

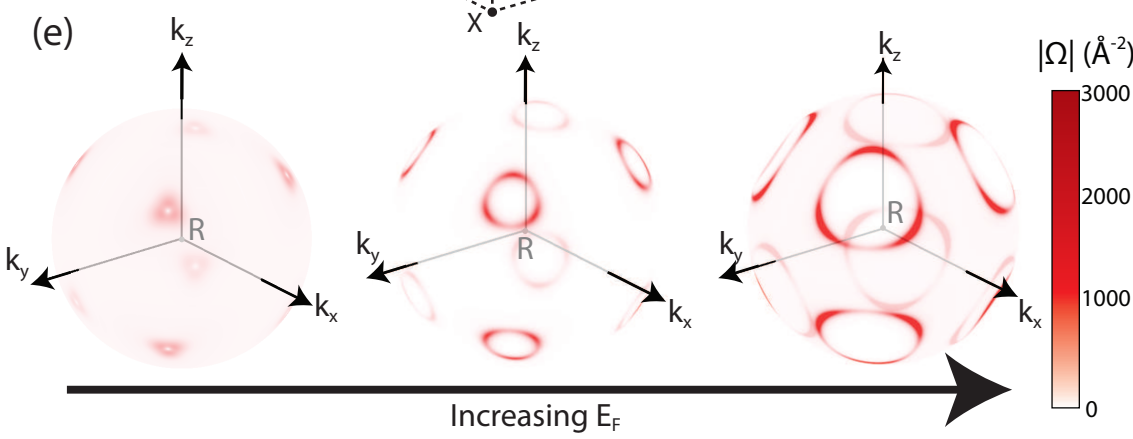

Figure 1: Illustration of quasi-symmetry. (a) If a crystalline symmetry operation commutes with the Hamiltonian system, the band crossing point is therefore symmetry-protected. (b) In this case the Hamiltonian itself does not commute with the symmetry operation yet its first order perturbation does. This lead to the situation where though the band crossing is numerically avoided but the resulted gap size is negligible for the physical properties of the system. This type of symmetry is called quasi-symmetry. (c) If the symmetry operation does not commute with the Hamiltonian to any order of perturbation of the system, the crossing is not protected by any symmetry. This will result in a sizeable gap and lost of topological character. (d) Quasi-symmetry and crystalline-symmetry protected degenerate planes. (e) The Berry curvature distribution of the $1^{-}$-band [see Fig. 2(e)] at different Fermi energy calculated from the model Hamiltonian Eq. (1). 

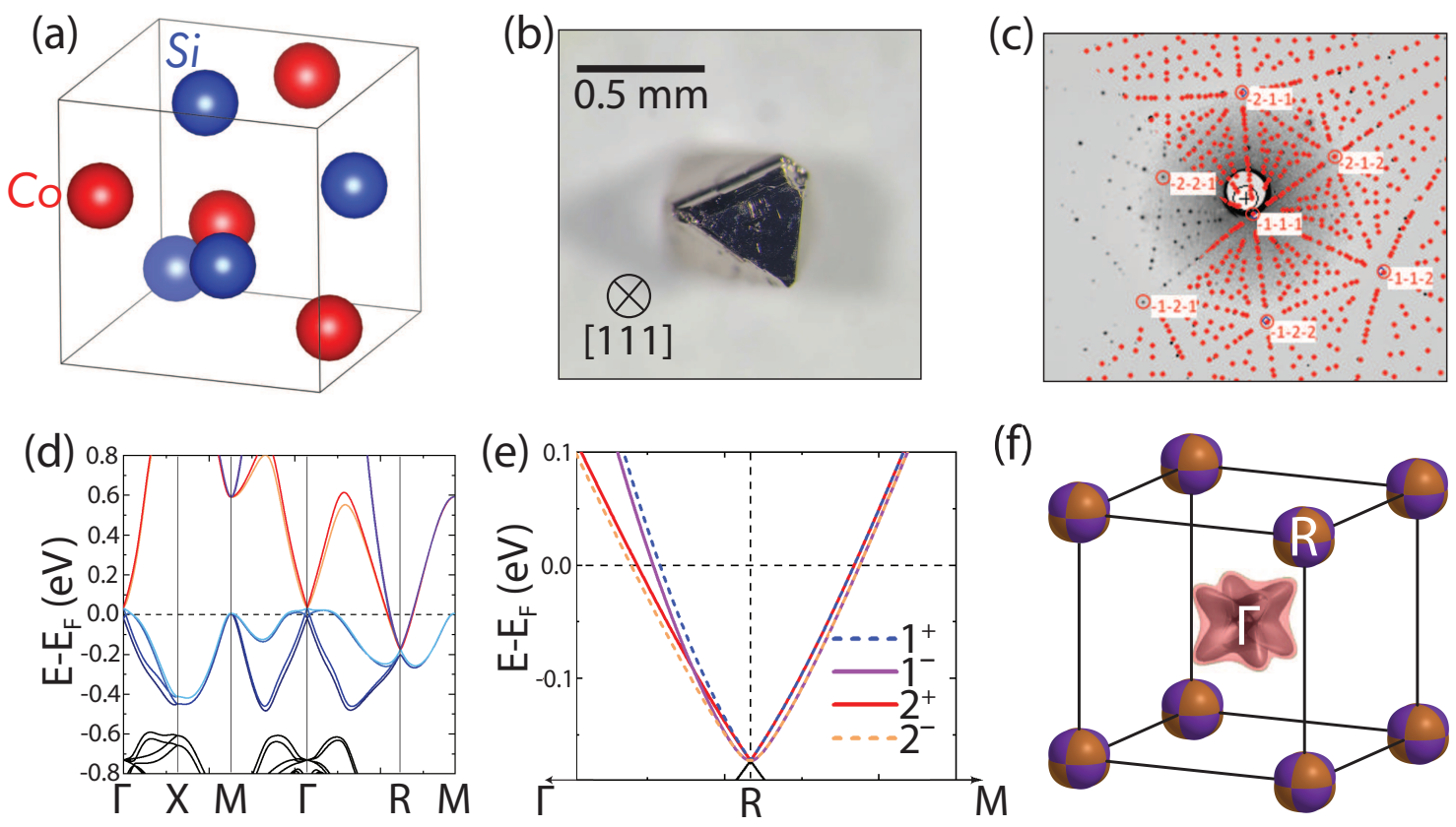

$(\mathrm{f})$
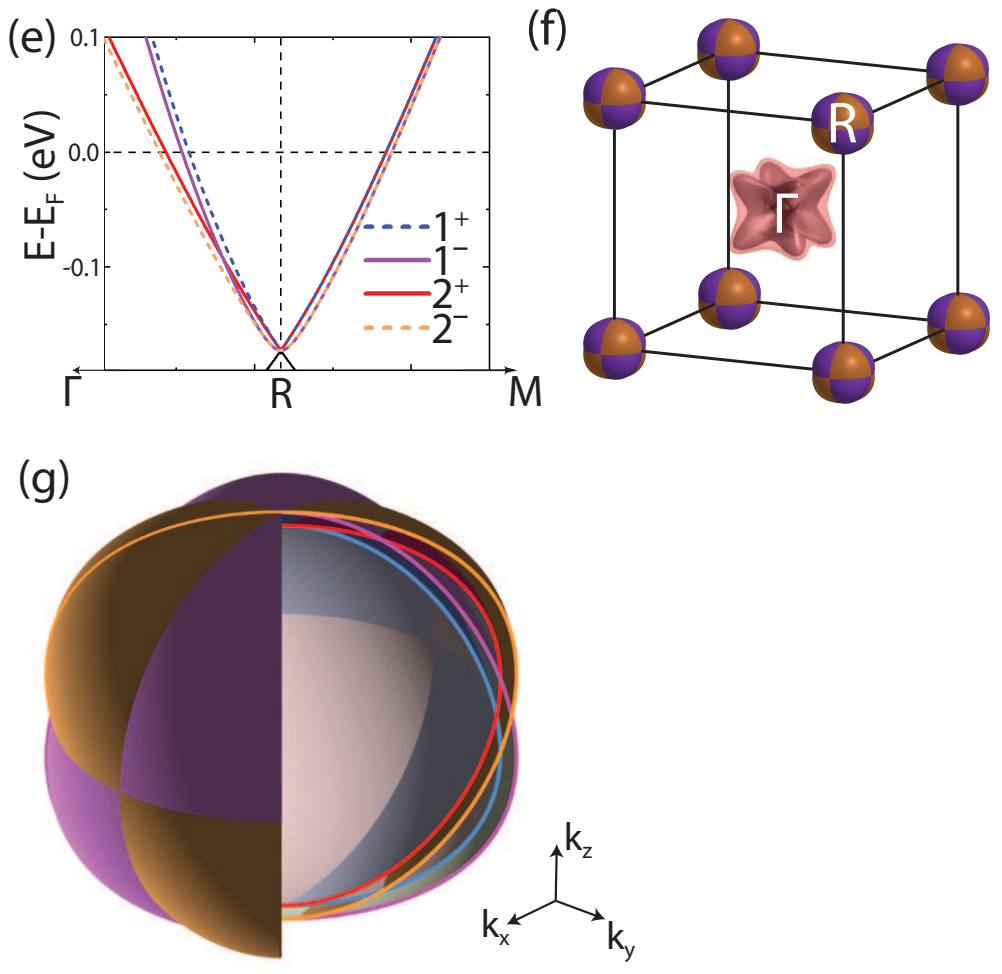

Figure 2: (a) Crystal structure of $\mathrm{CoSi}$. Co and $\mathrm{Si}$ atoms are presented as red and blue spheres respectively. (b) Optical microscope image of a CoSi single crystal sample. (c) Laue diffraction pattern of the grown CoSi single crystal, superimposed with a theoretically simulated one confirming high crystalline quality. (d) Ab-initio-calculated band structure of CoSi. (e) Enlarged section of band structure around R-point. Here 1/2 denotes orbital character while $+/$ - stands for the spin character of the band. (f) 3D view of all Fermi surfaces, which are centered around either R- or $\Gamma$ - point of the Brilloiun zone. (g) 3D view of Fermi surfaces centered around R-point with a quadrant cut. 
(a)

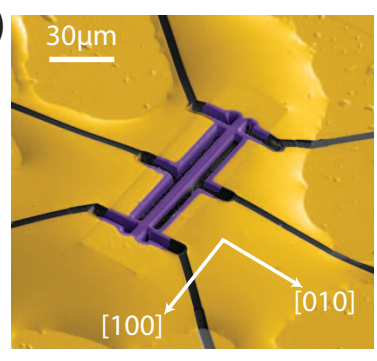

(b)

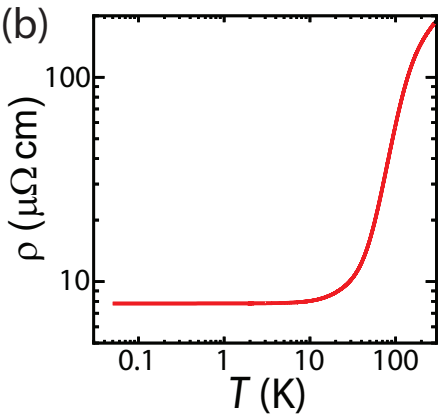

(c)

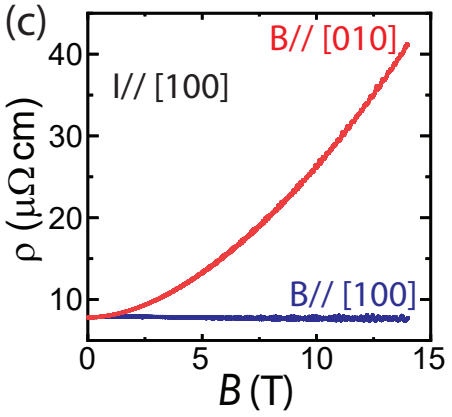

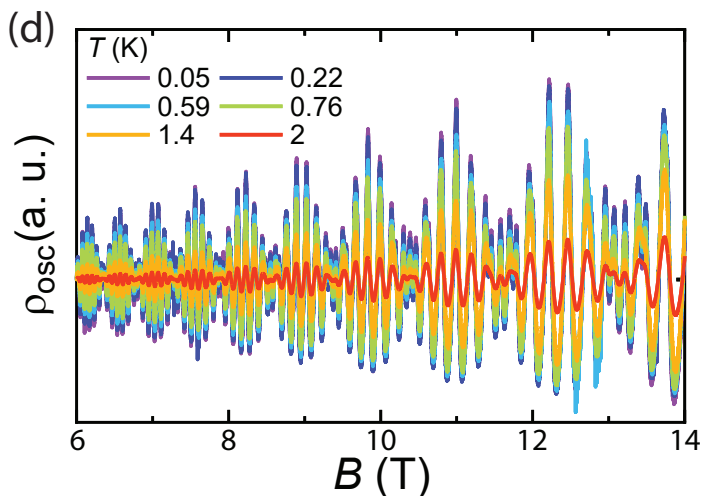
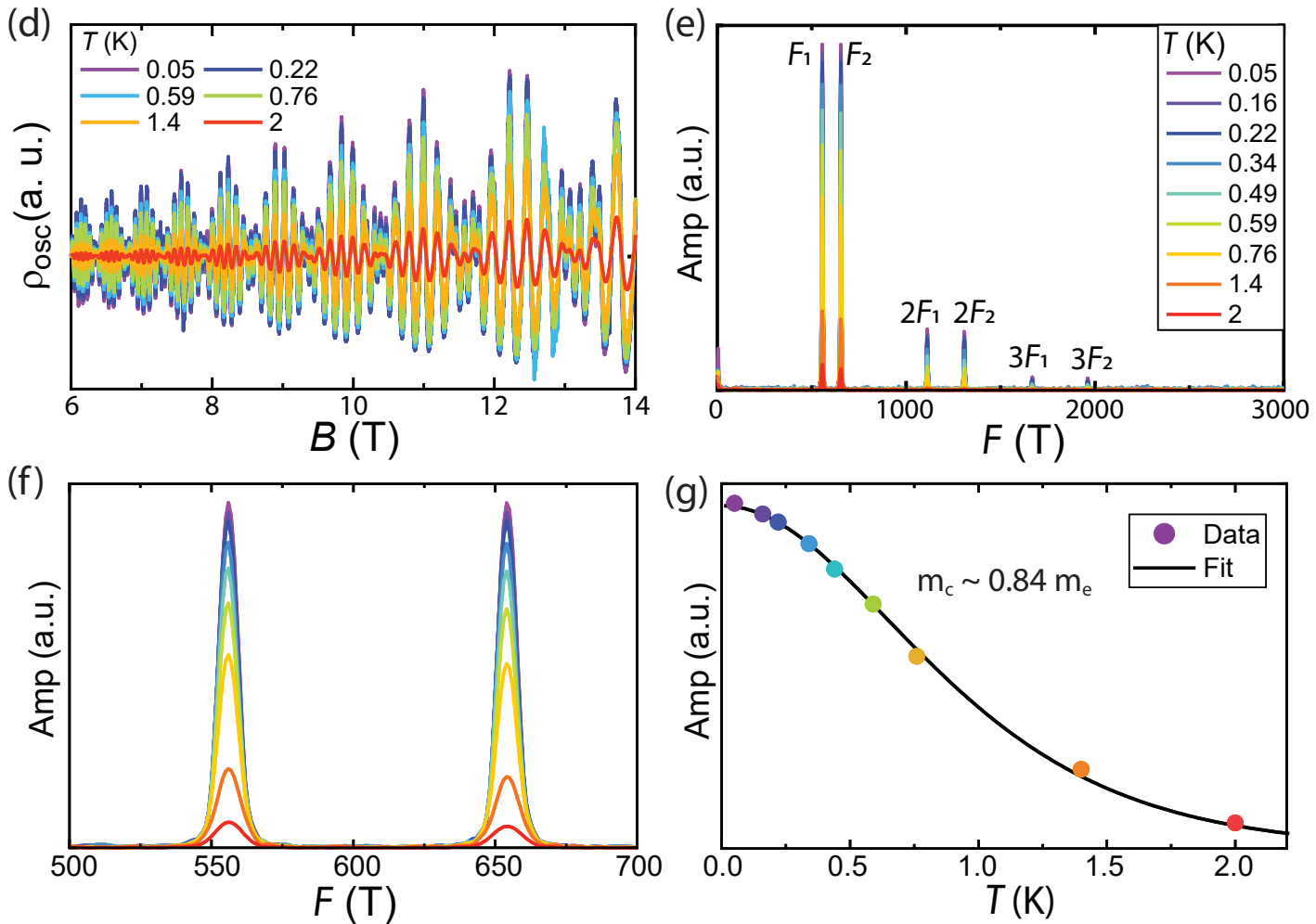

Figure 3: (a) Scanning electron microscope image of CoSi microdevice. A long bar along [100] direction with a 6 by $6 \mu \mathrm{m}^{2}$ cross section is fabricated by FIB. (b) Temperature dependence of resistivity measured from $300 \mathrm{~K}$ to $50 \mathrm{mK}$. (c) Transverse and longitudinal magnetoresistivity as a function of magnetic field. (d) Temperature-dependent $\mathrm{SdH}$ oscillations with field applied along [100] axis. Here $\rho_{o s c}=\Delta \rho / \rho_{B G}$, with $\Delta \rho$ the oscillatory part of the magnetoresistivity, and $\rho_{B G}$ the background obtained from a $3^{r d}$-order polynomial fit to the magnetoresistivity. (e) Fast-Fourier-transformation spectrum of the $\mathrm{SdH}$ oscillations presented in (d) with the field window of 3 to $14 \mathrm{~T}$. Two main peaks, as well as their higher-harmonic components, can be clearly observed. The suppression of peak amplitude with increasing temperature is due to the thermal damping effect. (f) Lifshitz-Kosevich fit to the temperature dependence of cyclotron mass. The fitting yields a cyclotron mass $m_{c} \sim 0.84 m_{e}$, comparable to the previously reported values (34-36). 

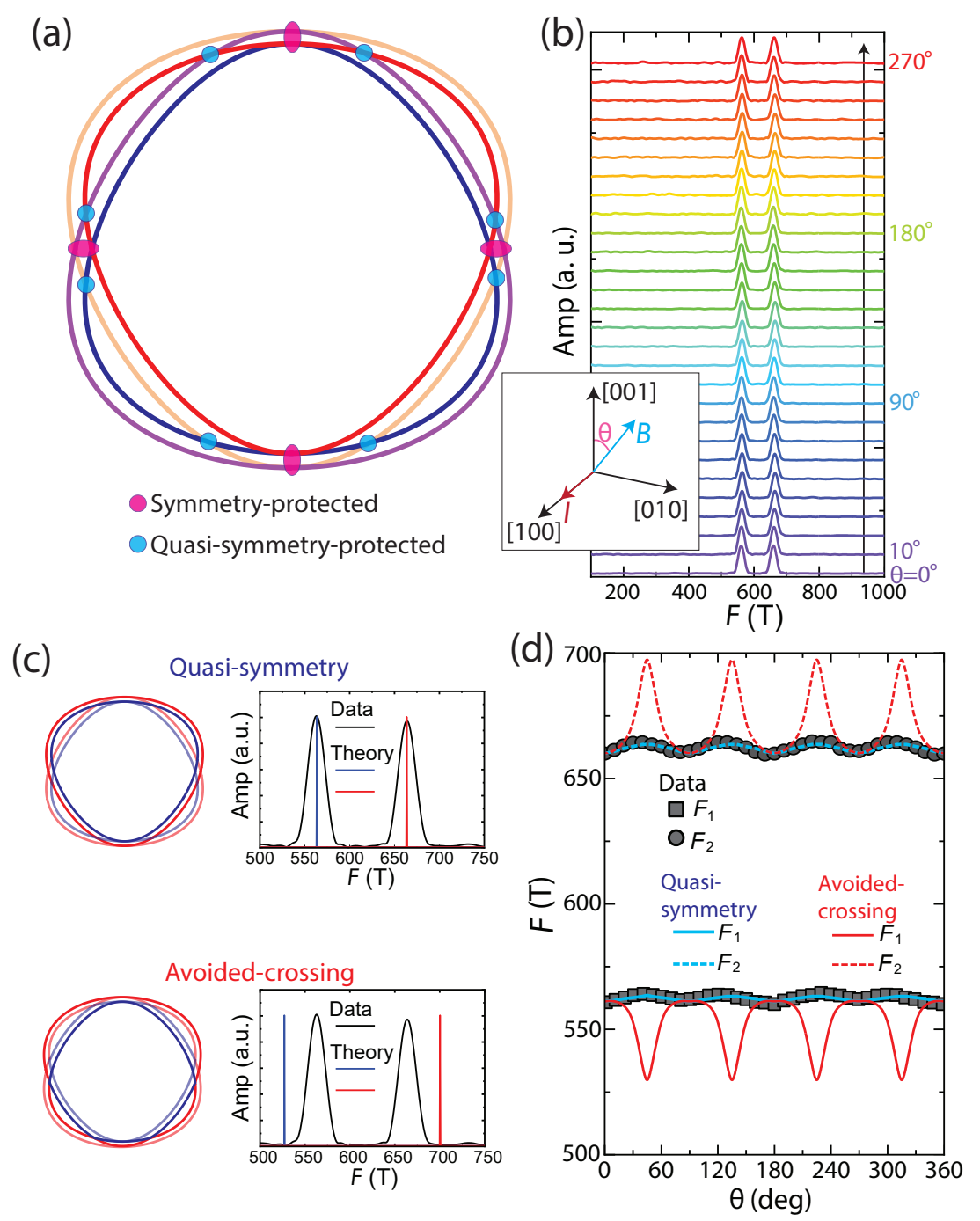

Figure 4: (a) (011)-orbit with symmetry-protected (pink) and quasi-symmetry protected (blue) degenerate points encircled. The symmetry-protected degenerate points always locate at the high symmetry plane while the quasi-symmetry protected crossings are positioned at arbitrary low-symmetry point. (b) Fast-fourier-transformation spectrum of angle-dependent quantum oscillations measured at $T=2 \mathrm{~K}$. Here the magnetic field is rotated within (100) plane and the angle is defined between the field direction and [001] axis. (c) Landau orbits and corresponding FFT spectrum for Quasi-symmetry and avoided-crossing scenarios. It is clear that only the quasi-symmetry scenario reproduces FFT peaks that match perfectly well with the experimental data. For the avoided crossing case the predicted frequency is far off. (d) Summary of angular dependence of oscillation frequencies. The contrast between two different scenario again clearly demonstrates that quasi-symmetry is the only option to explain the experimental results of nearly angle-independent oscillation frequencies. 


\section{Supplementary Files}

This is a list of supplementary files associated with this preprint. Click to download.

- HiddenQSymmetrySI.pdf 